\title{
Low temperature synthesis of anacardic-acid-capped cadmium chalcogenide nanoparticles
}

\author{
Sixberth S. Mlowe $\cdot$ Rajasekhar R. Pullabhotla • \\ Egid E. Mubofu • Faustine F. Ngassapa • \\ Neerish N. Revaprasadu
}

Received: 29 November 2013/Accepted: 14 May 2014/Published online: 8 June 2014

(c) The Author(s) 2014. This article is published with open access at Springerlink.com

\begin{abstract}
We report the low temperature synthesis of $\mathrm{CdE}(\mathrm{E}=\mathrm{S}$, Se and $\mathrm{Te})$ semiconductor nanoparticles capped by anacardic acid, an environmentally benign naturally occurring capping ligand. The cadmium chalcogenide nanoparticles were synthesized by reacting the reduced chalcogen powder with cadmium chloride to form the corresponding bulk material which was then dispersed in tri- $n$-octylphosphine (TOP) and thermolysed in anacardic acid. The low temperature of the reaction and the use of a naturally occurring oil solvent make this a relatively green synthetic route. The optical properties of the anacardicacid-capped cadmium chalcogenide particles show evidence of quantum confinement. The particles were characterized by X-ray diffraction and electron microscopy techniques.
\end{abstract}

Keywords Anacardic acid C CdE - Nanoparticles . Optical properties $\cdot$ Electron microscopy

\footnotetext{
S. S. Mlowe $(\bowtie) \cdot$ R. R. Pullabhotla · E. E. Mubofu

F. F. Ngassapa · N. N. Revaprasadu

University of Zululand, Private Bag X1001,

Kwadlangezwa 3886, South Africa

e-mail: sam.nanochem@gmail.com

R. R. Pullabhotla

e-mail: rajasekharp@yahoo.com

E. E. Mubofu

e-mail: ebmubofu@gmail.com

F. F. Ngassapa

e-mail: ngassapaf@yahoo.com

N. N. Revaprasadu

e-mail: RevaprasaduN@unizulu.ac.za
}

\section{Background}

The ability to fabricate structures at the nanoscale level has advanced in the field of material science. Semiconducting quantum dots have received much attention because of their tunable properties [1-5]. There has been considerable progress in the synthetic protocols, techniques and sample analysis of these nanosized materials. Two broad approaches have emerged to fabricate materials at this level: a top-down approach which the size and dimensionality of solid matter has gradually been reduced, and a bottom-up approach in which quantum dots are viewed as enormously large molecules [5, 6].

Cadmium chalcogenide (CdS, CdSe and CdTe) nanomaterials present enormous industrial interest in applications such as biomedical labeling reagents [7, 8], photovoltaic and optoelectronic devices [9-12]. The unique optical properties of these nanomaterials are dictated by their structure, shape and size, and the possibilities for band gap tailoring and tunable luminescence [2, 13, 14]. However, despite a remarkable amount of research, morphological control in semiconductor nanoparticle synthesis is still quite difficult. For semiconductor nanoparticles, the size- and shape-dependent optical properties of these nanomaterials have been found to be directly reliant on the resulting morphology [15].

To date many fabrication methods have been developed to synthesize high quality cadmium chalcogenide nanoparticles $[14,16]$. This has provided a great opportunity of using these materials in ways that exploit their novel properties in their various potential applications. The high temperature thermolysis route is one of the pioneering routes to cadmium chalcogenide nanoparticles. In this method dimethyl cadmium was used as the cadmium source [14] and tri- $n$-octylphosphine chalcogenide (TOPE) 
as the chalcogenide source at high reaction temperatures. While much of the fundamental studies on these materials was on materials synthesized using this route, it was deemed not suitable for large scale production of nanomaterials due to the high reaction temperatures and the toxic and pyrophoric nature of the metal alkyl. To avoid these drawbacks, three alternative methods were developed. Firstly, design of low temperature processing methods [17, 18], replacement of $\left(\mathrm{CH}_{3}\right)_{2} \mathrm{Cd}$ as $\mathrm{Cd}$-metal source by $\mathrm{CdO}$ $[16,19]$ and other $\mathrm{Cd}$ metal salts $[20,21]$ and lastly, the use of safe, cheaper, natural and more air stable coordinating solvents [17, 22, 23] such as olive oil have produced stable semiconductor nanoparticles.

Recently we reported the synthesis of lead chalcogenide nanoparticles capped by anacardic acid, a naturally occurring oil which is extracted from cashew nut shell liquid (CNSL) [24]. We have used the same synthetic procedure to synthesize cadmium chalcogenides (CdE) capped by anacardic acid. The potential of the work lies in using the greener solvent in the synthesis of cadmium chalcogenide nanoparticles at a relatively low temperature.

\section{Methods}

All chemicals used were of analytical grade. Selenium powder, tellurium powder, sulfur powder, sodium borohydride $\left(\mathrm{NaBH}_{4}\right)$, cadmium chloride, deionized water, methanol, toluene and tri- $n$-octylphosphine (TOP) were purchased from Sigma-Aldrich. The anacardic acid was isolated from solvent extracted cashew nut shell liquid (CNSL), using the method described by Paramashivappa et al. [25] with slight modifications reported by Lucio et al. [26].

The chalcogen powder was reduced to the corresponding ion by adding $0.32 \mathrm{mmol}$ of chalcogen powder to $20.0 \mathrm{~mL}$ of deionized water in a three neck flask at room temperature. A 0.79 mmol solution of sodium borohydride was added to the flask contents and the flask was purged with nitrogen flow to create an inert atmosphere. The reduction reaction was carried out for $2 \mathrm{~h}$ with continuous stirring and with the flow of $\mathrm{N}_{2}$ gas. A $0.32 \mathrm{mmol}$ solution of the cadmium salt was added to the reduced ion solution and stirred for $5 \mathrm{~min}$, followed by the addition of excess methanol to form the bulk $\mathrm{CdE}(\mathrm{E}=\mathrm{S}, \mathrm{Se}$ and $\mathrm{Te})$ which was isolated by centrifugation and dispersed in $6.0 \mathrm{~mL}$ of TOP. The resultant CdE-TOP mixture was then injected into $6.0 \mathrm{~g}$ of anacardic acid, pre-heated to $140{ }^{\circ} \mathrm{C}$. The reaction temperature was kept for $2 \mathrm{~h}$. The reaction contents were cooled to room temperature, followed by the addition of excess methanol to flocculate the nanoparticles. The anacardic-acid-capped CdE nanoparticles were isolated after centrifugation. The resultant nanoparticles were dissolved in toluene solvent for further characterization.

A Varian Cary 50 Conc UV-Visible spectrophotometer and Perkin-Elmer LS 55 Luminescence spectrometer were used to measure the absorption and photoluminescence properties of the particles, respectively. The crystalline phase was identified by X-ray diffraction (XRD), at a scanning rate of $0.05 \mathrm{~min}^{-1}$ in a $2 \theta$ range from $20^{\circ}$ to $80^{\circ}$, using a Bruker AXS D8 diffractometer equipped with nickel filtered Co $\mathrm{K} \alpha$ radiation $(\lambda=1.5418 \AA)$ at $40 \mathrm{kV}$. A JEOL 1010 TEM with an accelerating voltage of $100 \mathrm{kV}$, Megaview III camera, and Soft Imaging Systems iTEM software was used to determine the morphology.

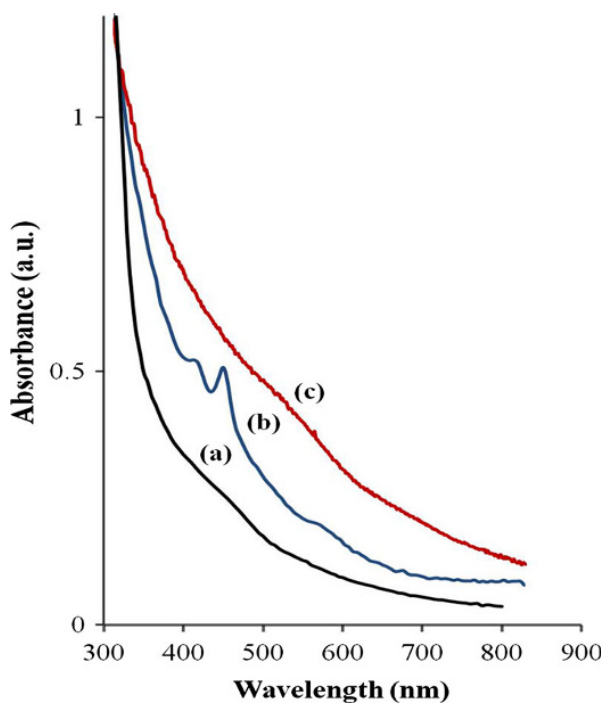

Fig. 1 UV-Visible absorption spectra of anacardic acid capped $a \mathrm{CdS}, b \mathrm{CdSe}$ and $c$ CdTe nanoparticles synthesized at $140{ }^{\circ} \mathrm{C}$

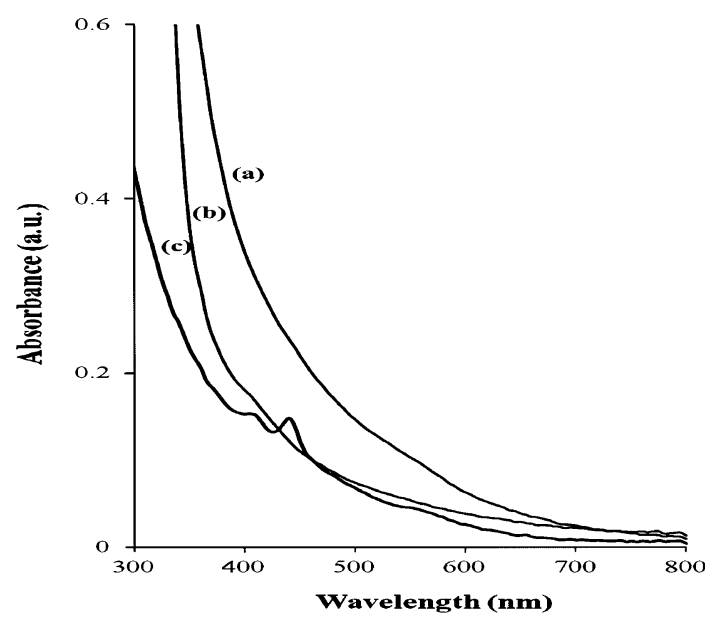

Fig. 2 UV-Visible absorption spectra of anacardic-acid-capped CdSe nanoparticles synthesized at $140{ }^{\circ} \mathrm{C}$ at different time intervals of $a 30 \mathrm{~min}, b 1 \mathrm{~h}$ and $c 2 \mathrm{~h}$ 


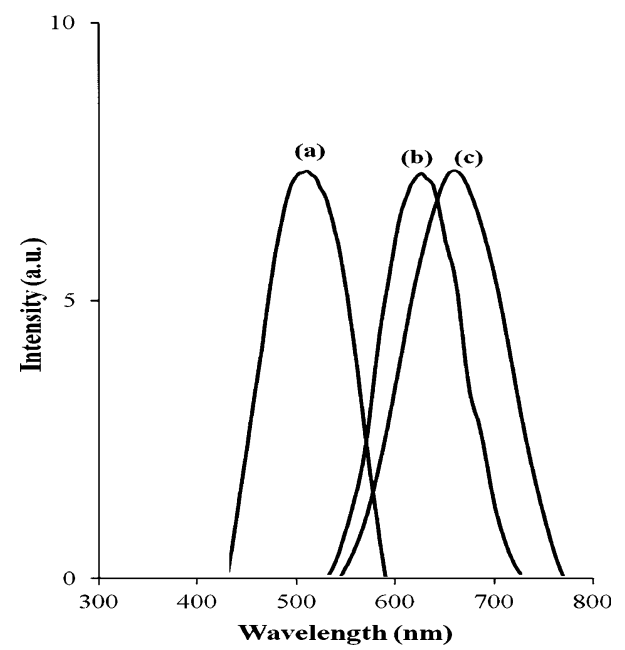

Fig. 3 Photoluminescence spectra of anacardic acid capped $a$ CdS, $b$ CdSe and $c$ CdTe nanoparticles synthesized at $140{ }^{\circ} \mathrm{C}$

HRTEM was done with a JEOL 2010 transmission electron microscope operated at an accelerating voltage of $200 \mathrm{kV}$.

\section{Results and discussion}

Optical properties by UV-Vis and photoluminescence analysis

Quantum size effects have been observed experimentally for many nanocrystalline semiconductor nanoparticles [27]. The absorption spectrum of a semiconductor nanoparticle provides an accessible and straight forward method for the evaluation of quantum size effects. Figure 1 shows the room temperature $\mathrm{UV}-\mathrm{Vis}$ absorption spectra of the anacardic-acid-capped $\mathrm{CdS}, \mathrm{CdSe}$ and $\mathrm{CdTe}$ nanoparticles thermolysed at $140{ }^{\circ} \mathrm{C}$. The absorption band edge for the anacardic-acid-capped $\mathrm{CdS}$ is observed at $505 \mathrm{~nm}$ $(2.46 \mathrm{eV})$, without any distinct excitonic features. The $\mathrm{CdSe}$ spectrum shows three distinct resolved electronic transitions, which could be assigned to the excitonic absorption features of nanosized CdSe [28] as predicted theoretically [29]. These transitions between any two electronic levels are seen as discrete peaks in the optical spectra, evidence of a quantum confined system [30]. Previous studies have also showed the same trend for CdSe
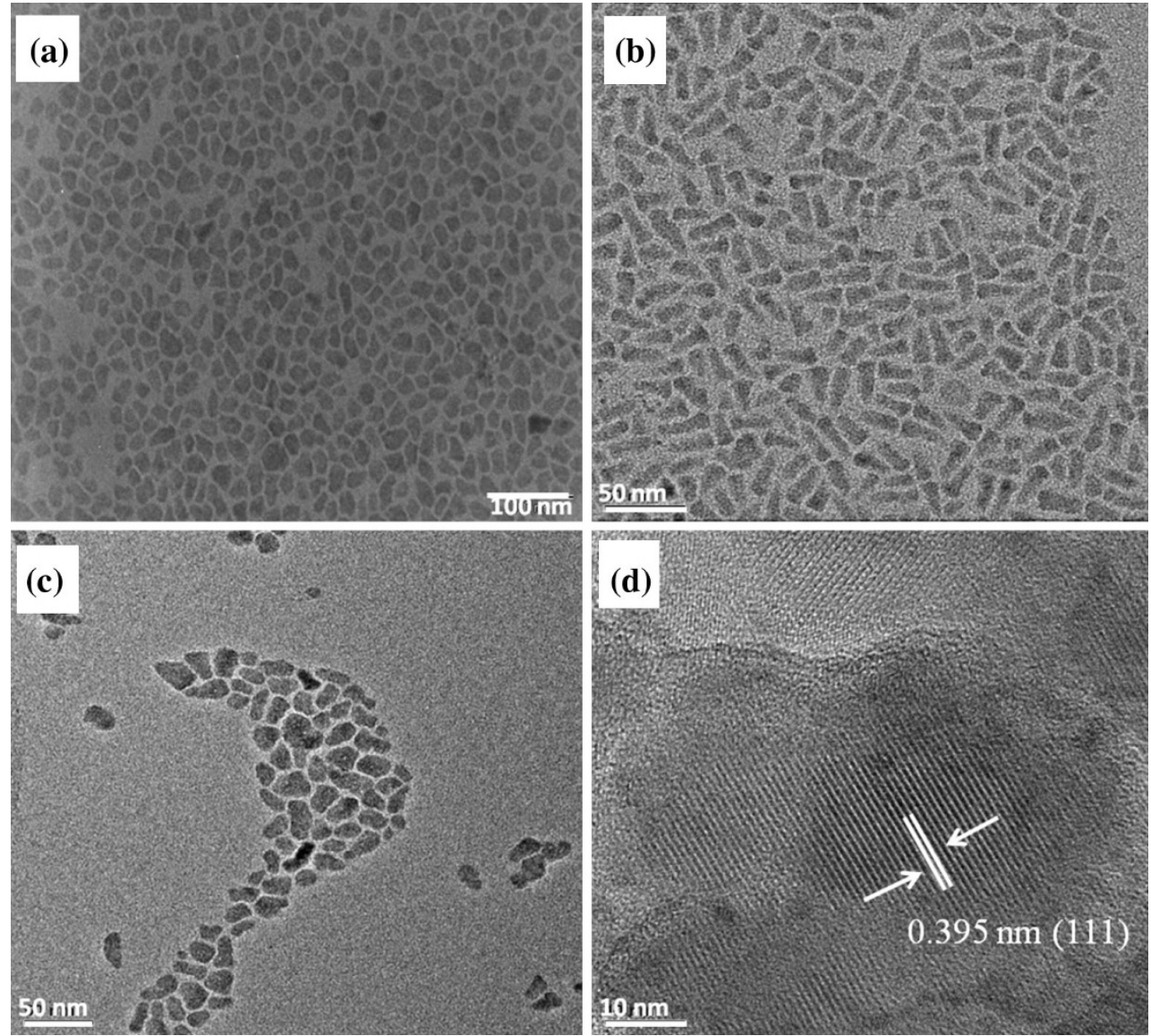

Fig. 4 TEM images of anacardic acid capped a CdS, b CdSe and $\mathbf{c}$ CdTe, and $\mathbf{d}$ HRTEM image of CdTe nanoparticles synthesized at $140{ }^{\circ} \mathrm{C}$ 

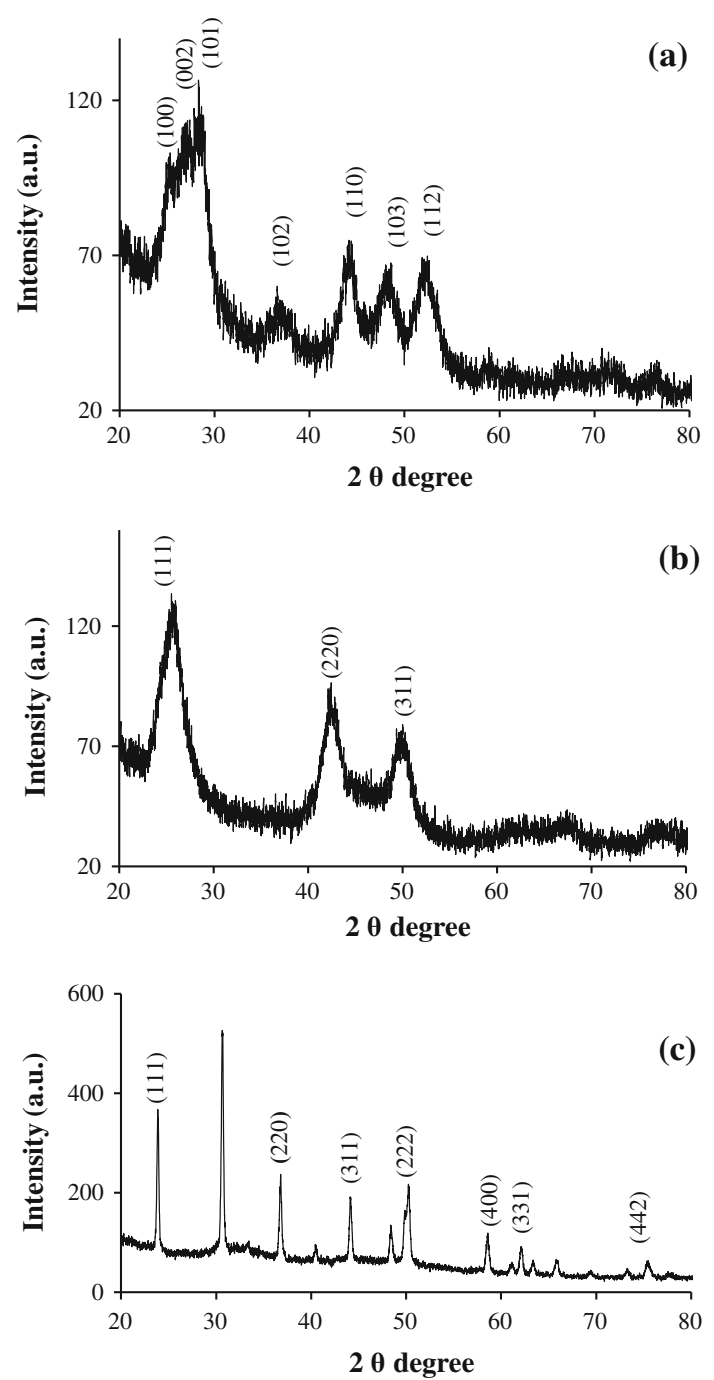

Fig. 5 Powder X-ray diffraction spectra of anacardic acid capped a CdS, b CdSe and c CdTe nanoparticles

nanoparticles thermolysed via the organometallic route [31]. There is an excitonic shoulder at $547 \mathrm{~nm}$, a distinct excitonic peak at $450 \mathrm{~nm}$ and a band edge at $620 \mathrm{~nm}$ $(2.0 \mathrm{eV})$. The anacardic-acid-capped CdTe shows a band edge at $720 \mathrm{~nm}(1.72 \mathrm{eV})$. All three samples show blue shifts in the absorption band edges in relation to their bulk band gaps implying the quantum confinement effect of these particles.

Figure 2 shows the combined absorption spectra of the 30, 60 and 120 min samples of the anacardic-acid-capped CdSe nanoparticles. There is no pronounced shift in the band gap as the reaction time increases from 30 to $120 \mathrm{~min}$ other than evolution of pronounced excitonic features observed for the sample collected after $120 \mathrm{~min}$. This result revealed that the size of CdSe nanocrystals gradually increased as reaction time prolonged, probably because of the low temperature of reaction. The photoluminescence spectra of anacardic-acid-capped CdS, CdSe and CdTe nanoparticles synthesized at $140{ }^{\circ} \mathrm{C}$ is shown in Fig. 3. For $\mathrm{CdSe}$, the emission peak $(637 \mathrm{~nm})$ is red shifted to that of absorption maximum $(620 \mathrm{~nm})$ which can be attributed to a combination of relaxation into shallow trap states and the size distribution [32]. All three PL spectra justify highly monodispersed samples and narrow emission line widths indicating growth of nanoparticles with few electronic defect sites as a result of the efficiency of anacardic acid to electronically passivate the nanoparticles.

\section{TEM and HRTEM analysis}

The morphology of the cadmium chalcogenide nanoparticles was studied by transmission electron microscopy (TEM) and high resolution TEM. Figure 4a shows the TEM image of the anacardic-acid-capped CdS nanoparticles synthesized at $140{ }^{\circ} \mathrm{C}$. The particles are anisotropic ranging from oblate to elongated shaped particles with an average size of $14.02 \pm 2.4 \mathrm{~nm}$. The CdSe nanoparticles and CdTe nanoparticles show similar morphology to that of CdS. The CdSe particles are slightly more elongated than the CdS particles, with an average length of $28.26 \pm 3.8 \mathrm{~nm}$ and width of $8.3 \pm 1.3 \mathrm{~nm}$ (Fig. 4b). The CdTe particles (Fig. 4c) were more oblate in shape with an average size of $14.90 \pm 2.23 \mathrm{~nm}$. The HRTEM image (Fig. 4d) of a single particle shows distinct lattice fringes with the interplanar distance of $3.83 \AA$, corresponding to the (111) plane of cubic CdTe. The assembly of all the anacardic-acid-capped CdS, CdSe and CdTe nanoparticles is in a well ordered, mosaic-type arrangement of particles (Fig. 4). There is almost an equal inter-particle distance of approximately $3 \mathrm{~nm}$ for the CdS, CdSe and CdTe particles as observed from the TEM images.

XRD analysis

Figure 5a shows the powder X-ray diffraction pattern of the anacardic-acid-capped CdS nanoparticles. The XRD pattern shows the presence of (100), (002), (101), (102), (110) and (103) reflection planes of the hexagonal phase of CdS. The average crystallite size was calculated to be $14.37 \mathrm{~nm}$, using the Scherrer formula $[33,34]$ corroborating the size determined by TEM.

The X-ray diffraction peaks (Fig. 5b) of the as-synthesized anacardic-acid-capped $\mathrm{CdSe}$ nanoparticles show three distinct peaks at $2 \theta$ values of $25.48^{\circ}, 42.34^{\circ}$ and $50.10^{\circ}$ corresponding to the (111), (220) and (311) planes of cubic CdSe, respectively. The high intensity of (111) peak indicates that the elongated particles have a large number of (111) planes thus making that peak the dominant reflection in the first diffraction feature [35]. The anacardic-acid-capped CdTe (Fig. 5c) also has a dominant cubic 
structure, which is also the dominant crystal phase of bulk CdTe. The reflections are indexed to the (111), (220), and (311) planes of the cubic CdTe [36]. The presence of the (222), (400) and (311) planes further supports the existence of cubic structure of CdTe.

\section{Conclusions}

Cadmium chalcogenide (CdE) nanoparticles have been successfully synthesized using anacardic acid as a coordinating solvent. The UV-Vis and PL studies confirmed that the CdE nanoparticles undergo quantum confinement effects. The TEM studies reveal a mosaic-like pattern for all three samples. The results reveal that anacardic acid could be an effective and potential capping agent in the synthesis of metal chalcogenide nanoparticles. The method used an inexpensive and non-toxic naturally occurring acid as a capping agent which proved to be a good choice for high quality, monodispersed nanoparticles.

Conflict of interest The authors declare that they have no competing interests.

Author's contributions SM carried out the experiments and drafted the manuscript. VSRP carried out XRD, TEM and HRTEM analysis. EBM, FNN and NR developed the conceptual framework and supervised the whole work. All authors read and approved the final manuscript.

Open Access This article is distributed under the terms of the Creative Commons Attribution License which permits any use, distribution, and reproduction in any medium, provided the original author(s) and the source are credited.

\section{References}

1. Lane, N.: The grand challenges of nanotechnology. J. Nanopart. Res. 3, 95-103 (2001)

2. Alivisatos, A.P.: Semiconductor clusters, nanocrystals, and quantum dots. Science 271, 933-937 (1996)

3. Ciftja, O., Faruk, M.M.: Two-dimensional quantum-dot helium in a magnetic field: variational theory. Phys. Rev. B 72, 205334 (2005)

4. Harju, A., Sverdlov, V.A., Barbiellini, B., Nieminen, R.M.: Variational wave function for a two- electron quantum dot. Phys. B 255, 145-149 (1998)

5. Grundmann, M., Christen, J., Ledentsov, N.N., Böhrer, J., Bimberg, D., Ruvimov, S.S., Werner, P., Richter, U., Gösele, U., Heydenreich, J., Ustinov, V.M., Egorov, Y.A., Zhukov, E., Kop'ev, P.S., Alferov, Z.I.: Ultranarrow luminescence lines from single quantum dots. Phys. Rev. Lett. 74, 4043-4046 (1995)

6. Ciftja, O.: Understanding electronic systems in semiconductor quantum dots. Phys. Scr. 88, 058302 (2013). doi:10.1088/00318949/88/05/058302

7. Bruchez, M., Moronne, M., Gin, P., Weiss, S., Alivisatos, A.P.: Semiconductor nanocrystals as fluorescent biological labels. Science 281, 2013-2016 (1998)
8. Chan, W.C.W., Nie, S.M.: Quantum dot bioconjugates for ultrasensitive nonisotopic detection. Science 281, 2016-2018 (1998)

9. Wang, Y., Tang, Z.Y., Tan, S.S., Kotov, N.A.: Biological assembly of nanocircuit prototypes from protein-modified CdTe nanowires. Nano Lett. 5, 243-248 (2005)

10. Chen, J., Song, J.L., Sun, X.W., Deng, W.Q., Jiang, C.Y., Lei, W., Huang, J.H., Liu, R.S.: An oleic acid-capped CdSe quantumdot sensitized solar cell. Appl. Phys. Lett. 94, 153115 (2009)

11. Gao, M., Lesser, C., Kirstein, S., Möhwald, H., Rogach, A.L., Weller, H.: Electroluminescence of different colors from polycation/CdTe nanocrystal self-assembled films. J. Appl. Phys. 87, 2297-2302 (2000)

12. Greenham, N.C., Peng, X., Alivisatos, A.P.: Charge separation and transport in conjugated-polymer/semiconductor-nanocrystal composites studied by photoluminescence quenching and photoconductivity. Phys. Rev. B 54, 17628-17637 (1996)

13. Brus, L.E.: Electron-electron and electron-hole interactions in small semiconductor crystallites: the size dependence of the lowest excited electronic state. J. Chem. Phys. 80, 4403-4409 (1984)

14. Murray, C.B., Norris, D.J., Bawendi, M.G.: Synthesis and characterization of nearly monodisperse $\mathrm{CdE}(\mathrm{E}=\mathrm{S}, \mathrm{Se}, \mathrm{Te})$ semiconductor nanocrystallites. J. Am. Chem. Soc. 115, 8706-8715 (1993)

15. Manna, L., Scher, E.C., Alivisatos, A.P.: Synthesis of soluble and processable rod-, arrow-, teardrop-, and tetrapod-shaped CdSe nanocrystals. J. Am. Chem. Soc. 122, 12700-12706 (2000)

16. Peng, Z.A., Peng, X.: Formation of high-quality CdTe, CdSe, and $\mathrm{CdS}$ nanocrystals using $\mathrm{CdO}$ as precursor. J. Am. Chem. Soc. 123, 183-184 (2001)

17. He, R., Gu, H.: Synthesis and characterization of monodisperse CdSe nanocrystals at lower temperature. Colloids and surfaces. A Physicochem. Eng. Aspects 272, 111-116 (2006)

18. Dunpall, R., Nejo, A.A., Rajasekhar Pullabhotla, V.S., Opoku, A.R., Revaprasadu, N., Shonhai, A.: An in vitro assessment of the interaction of cadmium selenide quantum dots with DNA, iron, and blood platelets. IUBMB Life 64, 995-1002 (2012). doi:10. 1002/iub. 1100

19. Boatman, E.M., Lisensky, G.C., Nordell, K.J.: A safer, easier, faster synthesis for CdSe quantum dot nanocrystals. J. Chem. Educ. 82, 1697-1699 (2005)

20. Mntungwa, N., Rajasekhar Pullabhotla, V.S.R., Revaprasadu, N.: A facile route to shape controlled CdTe nanoparticles. Mater. Chem. Phys. 126, 500-506 (2011)

21. Maseko, N.N., Revaprasadu, N., Rajasekhar Pullabhotla, V.S.R., Karthik, R., O'Brien, P.: The influence of the cadmium source on the shape of CdSe nanoparticles. Mater. Lett. 64, 1037-1040 (2010)

22. Sapra, S., Rogath, A.L., Feldmann, J.: Phosphine-free synthesis of monodisperse CdSe nanocrystals in olive oil. J. Mater. Chem. 16, 3391-3395 (2006)

23. Patel, J., Mighri, F., Ajji, A.: Simple non-aqueous fabrication route for oleic acid capped luminescent cadmium sulphide quantum dots at relatively low temperature. Soft Nanosci. Lett. 1, 61-65 (2011)

24. Mlowe, S., Nejo, A.A., Rajasekhar Pullabhotla, V.S.R., Mubofu, E.B., Ngassapa, F.N., O’Brien, P., Revaprasadu, N.: Lead chalcogenides stabilized by anacardic acid. Mater. Sci. Semicond. Process. 16, 263-268 (2013)

25. Paramashivappa, R., Kumar, P.P., Vithayathil, P.J., Rao, A.S.: Novel method for isolation of major phenolic constituents from cashew (Anacardium occidentale L.) Nut Shell Liquid. J. Agric. Food Chem. 49, 2548-2551 (2001)

26. Lucio, P.L.L., Santos, C.O., Romeiro, L.A.S., Costa, A.M., Ferreira, J.R.O., Cavalcanti, B.C., Moraes, O.M., Costa-Lotufo, 
L.V., Pessoa, C., Santos, M.L.: Synthesis and cytotoxicity screening of substituted isobenzofuranones designed from Anacardic acids. Eur. J. Med. Chem. 45, 3480-3489 (2010)

27. Bawendi, M.G., Steigerwald, M.L., Brus, L.E.: The quantum mechanics of larger semiconductor clusters ("quantum dots"). Annu. Rev. Phys. Chem. 41, 477-496 (1990)

28. Ithuria, S., Dubertret, B.: Quasi 2D colloidal CdSe platelets with thicknesses controlled at the atomic level. J. Am. Chem. Soc. 130, 16504-16505 (2008)

29. Efros, A.L., Rosen, M., Kuno, M., Nirmal, M., Norris, D.J., Bawendi, M.G.: Band-edge exciton in quantum dots of semiconductors with a degenerate valence band: dark and bright exciton states. Phys. Rev. B 54, 4843-4856 (1996)

30. Yoffe, A.D.: Semiconductor quantum dots and related systems: electronic, optical, luminescence and related properties of low dimensional systems. Adv. Phys. 50, 1-208 (2001)

31. Norris, D.J., Efros, A.L., Rosen, M., Bawendi, M.G.: Size dependence of exciton fine structure in CdSe quantum dots. Phys. Rev. B 53, 16347-16354 (1996)
32. Bawendi, M.G., Carroll, P.J., Wilson, W.L., Brus, L.E.: Luminescence properties of CdSe quantum crystallites: resonance between interior and surface localized states. J. Chem. Phys. 96, 946-956 (1992)

33. Bandaranayake, R.J., Wen, G.W., Lin, J.Y., Jiang, H.X., Sorensen, C.M.: Structural phase behavior in II-VI semiconductor nanoparticles. Appl. Phys. Lett. 67, 831-833 (1995)

34. Patterson, A.L.: The Scherrer formula for X-Ray particle size determination. Phys. Rev. 56, 978-982 (1939)

35. Rogach, A.L., Kornowski, A., Gao, M., Eychmuller, A., Weller, H.: Synthesis and characterization of a size series of extremely small thiol-stabilized CdSe nanocrystals. J. Phys. Chem. B 103, 3065-3069 (1999)

36. Blokland, J.H., Claessen, V.I., Wijnen, F.J.P., Groeneveld, E., de Mello Donega, C., Vanmaekelberg, D., Meijerink, A., Maan, J.C., Christianen, P.C.M.: Exciton lifetime of CdTe nanocrystal quantum dots in high magnetic fields. Phys. Rev. B 83, 035304 (2011) 